\title{
Biaxial Constitutive Model of Active Coronary Media Based on Microstructural Information
}

\author{
Huan Chen ${ }^{1 *}$, Yunlong Huo ${ }^{2}$, Ghassan S. Kassab ${ }^{1,3}$ \\ ${ }^{1}$ Department of Biomedical Engineering, Indiana University Purdue University Indianapolis, Indianapolis, USA \\ ${ }^{2}$ Mechanics and Engineering Science, State Key Laboratory for Turbulence and Complex Systems, \\ College of Engineering, Peking University, Beijing, China \\ ${ }^{3}$ Department of Surgery, Cellular and Integrative Physiology, Indiana University Purdue \\ University Indianapolis, Indianapolis, USA \\ Email: *huanchen@iupui.edu
}

Received June 2013

\begin{abstract}
Detailed morphological data of vascular smooth muscle cells (VSMC) of coronary arteries were limited. The present study was to quantify dimensions and orientation of swine coronary VSMC and to develop a micro-structural constitutive model of active media. It was found that geometrical parameters of VSMC (length, width, spatial aspect ratio, and orientation) follow normal distributions, and VSMCs orientate towards the circumferential direction of vessels with oblique and symmetrical angles. A micro-structural model of media layer was developed to accurate $\longrightarrow$ accurately predict biaxial active responses of coronary arterial media, based on experimental measurements. The present morphological data base and micro-structural model lead to a better understanding of biomechanics of muscular vessels.
\end{abstract}

Keywords: Biaxial Constitutive Model; Cell Deformation; Morphology; Vascular Smooth Muscle Cell

\section{Introduction}

Many mechanical models of blood vessels suggested uniaxial active constitutive relations, based on one-dimensional length-tension relationships of vascular smooth muscle cells (VSMCs) in the circumferential direction of vessels. Some studies, however, observed significant biaxial responses (circumferential and axial) of blood vessels during vasoconstriction [1-5], which cannot be explained by previous uniaxial active models. The significant axial active response may be induced by VSMC helical orientation [6-9] and/or multi-axial active responses of individual VSMCs [2,3,10,11]. The arrangement of VSMCs, however, has been debated in literature. VSMCs were found to align in the circumferential direction in some studies [6,12-14], while they were observed to be oblique in others [6-9].

The present study aimed to describe biaxial vasoactivity by a micro-structural constitutive model of coronary artery, which accounts for geometrical and mechanical properties of individual collagen fibers, elastin fiber and VSMCs, respectively [11]. The morphology of VSMCs must be measured by using confocal microscopy, and four geometrical parameters (length, width, spatial aspect ratio, and orientation) were then quantified, and the de-

*Corresponding author. formation of individual VSMCs under various pressure distensions was also measured. Based on measured geometrical and deformation features of VSMC, a microstructural constitutive model, including muscle contraction was proposed to describe the biaxial contraction of coronary arterial media.

\section{Methods}

\subsection{Preparation of Samples}

6 hearts of healthy pigs were used in present study. The coronary arteries were dissected from hearts and cleaned carefully. 6 segments of $\sim 2 \mathrm{~cm}$ in length of each heart were prepared for 6 different distension pressures. A custom-made excess surface-area balloon tip catheter was inserted into each segment and distended to fully transmit pressure to the vessel lumen [15]. The distended segments were immersion-fixed in $0.8 \%$ methanol-free paraformaldehyde solution, the osmolarity of which was adjusted to $292 \pm 11 \mathrm{mOsm}$ ) with $\mathrm{pH}$ of 7.4, mimicking the osmolarity of normal extracellular fluids to avoid cell shrinkage and swelling [16]. The segment was then fixed at room temperature for $48 \mathrm{hrs}$ for later preparing of histological sections. $5 \times 5 \mathrm{~mm}^{2}$ cross-sections were then sectioned from the segment and the circumferential direction was marked after distention-fixation. The adven- 
titia was peeled off carefully from the vessel to expose media layer. The main protein type of the cellular cytoskeleton, F-actin, and the cellular nucleus were labeled (by Alexa Fluor 488 phalloidin and DAPI) to track geometries of VSMC under different distension loads. The sections were wet mounted on microscope slides using a glycerin-water mixture and then viewed under a FV1000MPE confocal microscope with a $60 \times 1$.1NA water immersion objective. Each segment was scanned at 3 different locations for each loading condition. The six loading conditions were considered: 1 ) zero-stress state (ZSS); 2) no-load state (0 mmHg distension); 3) $40 \mathrm{mmHg}$ distension; 4) $80 \mathrm{mmHg}$ distension; 5) $120 \mathrm{mmHg}$ distension; and 6) $160 \mathrm{mmHg}$ distension.

\subsection{Measurement of VSMC Geometry}

The geometry of VSMCs was determined by four parameters: length, width, spatial aspect ratio, and orientation angles (the circumferential direction of vessel was taken as $0^{\circ}$ and the axial direction as $90^{\circ}$ ). The orientation of the VSMC was measured using an automated algorithm, and dimensions of VSMCs were directly measured on confocal images [11]. The cell length and width were determined by the major and minor axes of each cell, and the aspect ratio, featuring the cell shape, was identified by the ratio of length to width of a given cell. The RGB image of the nucleus was first converted to a binary image, where the nucleus-containing pixels were clearly distinguished from the background by median filtering. The geometrical parameters of the nucleus were calculated automatically based on this binary image [11]. The measured result was expressed as the mean, mean \pm SD (standard deviation), of all measured cells in the images. The significance of the difference between the parameter under various loads was evaluated by a one-way ANOVA test (SigmaStat 3.5), while the results were considered statistically different when $\mathrm{P}<0.05$.

\subsection{Microstructure-Based Constitutive Model of Active Coronary Media}

The media segment was considered as a thin-walled elastic tube deformed in the circumferential and axial directions. A previously proposed structural constitutive model was used to describe the mechanical response of passive coronary media $[17,18]$, which contains isotropic inter-lamellar (IL) elastin networks and helically oriented collagen fibers:

$$
\left\{\begin{array}{l}
W_{E}(\mathbf{E})=\frac{f_{E}}{\pi} \int_{-\frac{\pi}{2}}^{\frac{\pi}{2}} w_{E}\left[e_{E}\left(\theta_{E}\right)\right] d \theta_{E} \\
W_{C}(\mathbf{E})=\frac{f_{C}}{2}\left\{w_{C}\left[e_{C}\left(\theta_{C}\right)\right]+w_{C}\left[e_{C}\left(-\theta_{C}\right)\right]\right\}
\end{array}\right.
$$

where $w_{E}$ and $w_{C}$ are the strain energy of elastin struts and collagen fibers, depending on uniaxial fiber strain $e_{s}$ ( $s=E, C$ denoting elastin and collagen, respectively). $\theta_{s}$ is the fiber orientation angle (corresponding unit vector denoted by $\overrightarrow{\mathbf{n}}^{s}$ ) and $f_{s}$ is the volume fraction. The fiber strain was determined by $e_{s}=\overrightarrow{\mathbf{n}}^{s} \cdot \mathbf{E} \cdot \overrightarrow{\mathbf{n}}^{s}$ with assuming affine deformation (i.e., a fiber is assumed to rotate and stretch in the same way as the bulk tissue). The linear stress-strain relation of elastin was considered as $\partial w_{E} / \partial e_{E}=k_{E} e_{E}$ while the nonlinear relation of collagen was considered as $\partial w_{C} / \partial e_{C}=k_{C} e_{C}^{N_{C}}$, with the material parameter $k_{E}$ representing the stiffness of the elastin, and $k_{C}$ and $N_{C}$ representing the stiffness and nonlinear parameter of collagen. The passive strain energy of the coronary media $W_{\text {passive }}$ was calculated by taking the sum of the strain energies of the elastin and collagen networks; i.e., $W_{\text {passive }}(\mathbf{E})=W_{E}(\mathbf{E})$ $+W_{C}(\mathbf{E})$, and the second Piola-Kirchhoff stress was accordingly determined by $\mathbf{S}_{\text {passive }}=\partial W_{\text {passive }}(\mathbf{E}) / \partial \mathbf{E}$.

The total strain energy of media is the sum of the active and passive contributions, and the active strain energy is dominantly contributed by active VSMC. Taking into consideration two families of helical VSMC with symmetrical angles, it can be given as:

$$
\begin{aligned}
& W_{\text {active }}\left(\lambda_{\text {VSMC }}, \lambda_{\text {VSMC }}^{\perp}\right) \\
= & \frac{f_{V S M C}}{2}\left\{w_{V S M C}\left\{\lambda_{\text {VSMC }}, \lambda_{\text {VSMC }}^{\perp}\right\}\left(\theta_{V S M C}\right)\right. \\
& +w_{V S M C}\left\{\lambda_{\text {VSMC }}, \lambda_{\text {VSMC }}^{\perp}\right\}\left(-\theta_{\text {VSMC }}\right)
\end{aligned}
$$

where $\theta_{V S M C}$ is the orientation angle of VSMC, $w_{V S M C}$ is multi-axial strain energy of a single VSMC and $f_{V S M C}$ is the volume fraction. A two-dimensional generalization of the uniaxial length-tension relation of active VSMC [19] was used to account for the multi-axial active response of VSMC [2,3]:

$$
\begin{aligned}
& w_{V S M C}\left(\theta_{V S M C}\right) \\
= & A C_{a c t}\left\{\operatorname { E r f } \left(\frac{\lambda_{V S M C}\left(\theta_{V S M C}\right)-b_{3}}{b_{1}}\right.\right. \\
& \left.\left.+\frac{\lambda_{V S M C}^{\perp}\left(\theta_{V S M C}\right)-b_{4}}{b_{2}}\right)+1\right\}
\end{aligned}
$$

where $\lambda_{\text {VSMC }}=\sqrt{\overrightarrow{\mathbf{n}}^{V S M C} \cdot\left(\mathbf{F}^{T} \cdot \mathbf{F}\right) \cdot \overrightarrow{\mathbf{n}}^{V S M C}}$ is the longitudinal stretch ratio of a VSMC (i.e., cell stretch), and $\lambda_{S M C}^{\perp}=\sqrt{\overrightarrow{\mathbf{n}}^{V S M C^{\prime}} \cdot\left(\mathbf{F}^{T} \cdot \mathbf{F}\right) \cdot \overrightarrow{\mathbf{n}}^{V S M C^{\prime}}}$ is the transversal stretch ratio ( $\mathbf{F}$ is deformation gradient). $\overrightarrow{\mathbf{n}}^{V S M C}$ and $\overrightarrow{\mathbf{n}}^{V S M C^{\prime}}$ are the longitudinal and transversal vectors, respectively. $A$ is the level of activation ( 0 is passive state and 1 is fully active), $C_{a c t}, b_{1}, b_{2}, b_{3}$ and $b_{4}$ are material constants, and $\operatorname{Erf(}()$ is the Gauss error function. Accordingly, 
the active stress of coronary media is determined as the derivatives of the strain energy function; i.e., $\mathbf{S}_{\text {active }}=$ $\partial W_{\text {active }}(\mathbf{E}) / \partial \mathbf{E}$. Therefore, the total stress is the sum of passive and active stresses.

We used biaxial data of coronary media obtained in our previous study [3] to determine material parameters in the structural model. The media was considered as a thin cylindrical shell and the 2nd Piola-Kirchhoff circumferential stress obtained by experimental measurement was determined by $S_{\theta \theta}^{\exp }=P r_{i} / h \lambda_{\theta}^{2}$, where $P$ is distension pressure, $r_{i}=\sqrt{r_{o}^{2}-A_{o} / \pi \lambda_{z}}$ is the inner radius in the loaded state, $r_{o}$ is the outer radius in the loaded state, $A_{o}$ is the wall area in a no-load state, and $h=r_{o}-r_{i}$ is the wall thickness in the loaded state. The axial stress was computed by

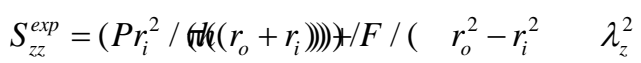

with $F$ presenting the axial force. The material parameters were determined by minimizing the square of the difference between the theoretical and experimental passive and active circumferential and axial 2nd Piola-Kirchhoff stresses [11]. The predicted strain-stress curves were thus determined by Equations (1)-(3).

\section{Results}

The probability distribution functions of cell geometrical parameters were found to follow to continuous normal or bimodal normal distributions (Table 1) at ZSS. The length of individual VSMC was $56.0 \pm 10.3 \mu \mathrm{m}$ which is larger than that of the nuclei $(15.0 \pm 4.7 \mu \mathrm{m})$ while their widths were similar $(3.9 \pm 0.7 \mu \mathrm{m}$ vs. $3.4 \pm 0.8 \mu \mathrm{m})$. The means of aspect ratio of the VSMC and the nuclei were $14.7 \pm 3.5$ and $4.6 \pm 1.7$, respectively. VSMC aligned off the circumferential direction of the vessel with a bimodal distribution with a mean angle of $\pm 18.7^{\circ} \pm 10.9^{\circ}$, consistent with the angle of the nucleus $\pm 19.9^{\circ} \pm 10.7^{\circ}$.

The VSMC of passive coronary media deformed significantly with an increase in distention pressure (Figure 1). The cells gradually shifted in the circumferential direction at elevated pressure. The VSMC were significantly

Table 1. The distribution of geometrical parameters of VSMC and the nucleus were fitted to a continuous normal distribution (or a bimodal normal distribution). $\mu$ is the mean of the distribution, $\sigma$ is standard deviation and $R^{2}$ presents goodness of fit.

\begin{tabular}{ccccccc}
\hline & \multicolumn{3}{c}{ Nucleus } & \multicolumn{3}{c}{ VSMC } \\
\hline Parameters & $\mu$ & $\sigma$ & $R^{2}$ & $\mu$ & $\sigma$ & $R^{2}$ \\
Length $(\mu \mathrm{m})$ & 15.0 & 4.7 & 0.96 & 56.0 & 10.3 & 0.98 \\
Width $(\mu \mathrm{m})$ & 3.4 & 0.8 & 0.85 & 3.9 & 0.7 & 0.93 \\
Aspect ratio & 4.6 & 1.7 & 0.80 & 14.7 & 3.5 & 0.88 \\
Orientation $\left({ }^{\circ}\right)$ & \pm 19.9 & 10.7 & 0.98 & \pm 18.7 & 10.9 & 0.92 \\
\hline
\end{tabular}

stretched in the axial direction and became morespindled with longer tails, while the nuclei did not significantly deform. Changes of geometries of VSMC and nuclei were fitted to a logarithmic function in Table 2. The length of VSMC raised significantly and nonlinearly $(\mathrm{P}<$ 0.05), with increase of distension pressure, while the length of the nuclei increased relatively slightly. The change of VSMC length increased sharply from the no-load state to $80 \mathrm{mmHg}$ distention and became plateaued at higher pressure. The widths of VSMC and the nuclei did not change significantly under all pressures $(\mathrm{P}>$ 0.05) (insignificant changes were not listed in Table 2). Accordingly, the aspect ratio of VSMC increased nonlinearly, while that of the nuclei did not change significantly $(\mathrm{P}>0.05)$. The orientation angles of VSMC and the nuclei decreased nonlinearly from the no-load state to $160 \mathrm{mmHg}$ distension $(\mathrm{P}<0.05)$.

The predicted reorientation of VSMC, determined by

$$
\begin{aligned}
& \theta_{V S M C}^{\prime}= \\
& \arccos \left(\cos \theta_{V S M C} \lambda_{\theta} / \sqrt{\cos ^{2} \theta_{V S M C} \lambda_{\theta}^{2}+\sin ^{2} \theta_{V S M C} \lambda_{z}^{2}}\right)
\end{aligned}
$$

( $\lambda_{z}=1.0$ in experimental study), was plotted in Figure 2(a), according to the affine deformation assumption. Correspondingly, the VSMC stretch ratio, determined by

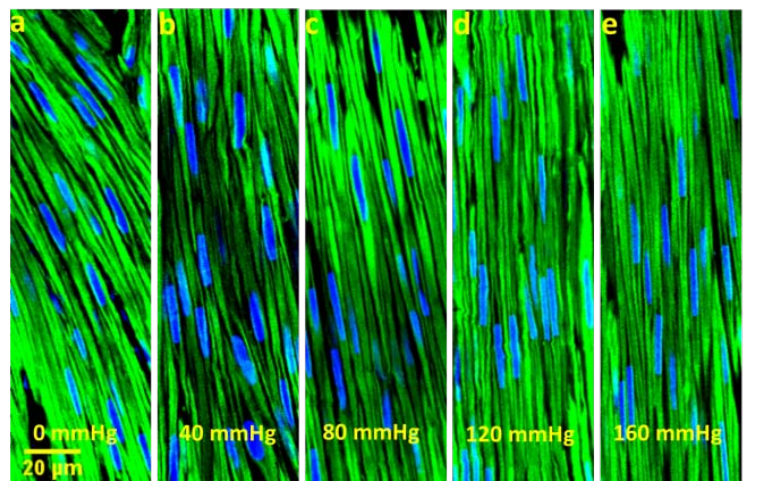

Figure 1. The deformed VSMC of coronary media under various distention pressures: The loading pressures in (a) (e) are: $0 \mathrm{mmHg}, 40 \mathrm{mmHg}, 80 \mathrm{mmHg}, 120 \mathrm{mmHg}, 160$ mmHg, respectively.

Table 2. Non-linear relations between geometrical parameters and distension pressures obtained by curve fitting to a logarithmic function: $y=a_{1}+a_{2} \log (x)$.

\begin{tabular}{ccccc}
\hline & Parameters & $a_{1}$ & $a_{2}$ & $R^{2}$ \\
\hline \multirow{3}{*}{ VSMC } & Length $(\mu \mathrm{m})$ & 60.2 & 7.7 & 0.95 \\
& Aspect Ratio & 17.4 & 1.8 & 0.90 \\
& Orientation $\left(^{\circ}\right)$ & 18.2 & -2.9 & 0.99 \\
& Stretch Ratio & 1.1 & 0.1 & 0.95 \\
& Length $(\mu \mathrm{m})$ & 16.1 & 0.9 & 0.81 \\
& Orientation $\left(^{\circ}\right)$ & 17.2 & -2.5 & 0.99 \\
\hline
\end{tabular}



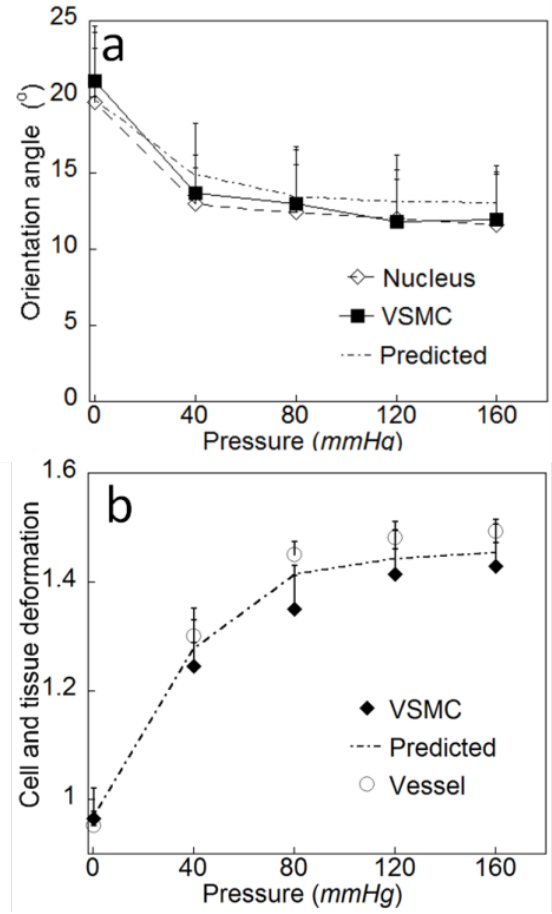

Figure 2. (a) Measured and predicted changes of the orientation angle of the VSMC and nucleus; (b) Measured and predicted VSMC and tissue deformation.
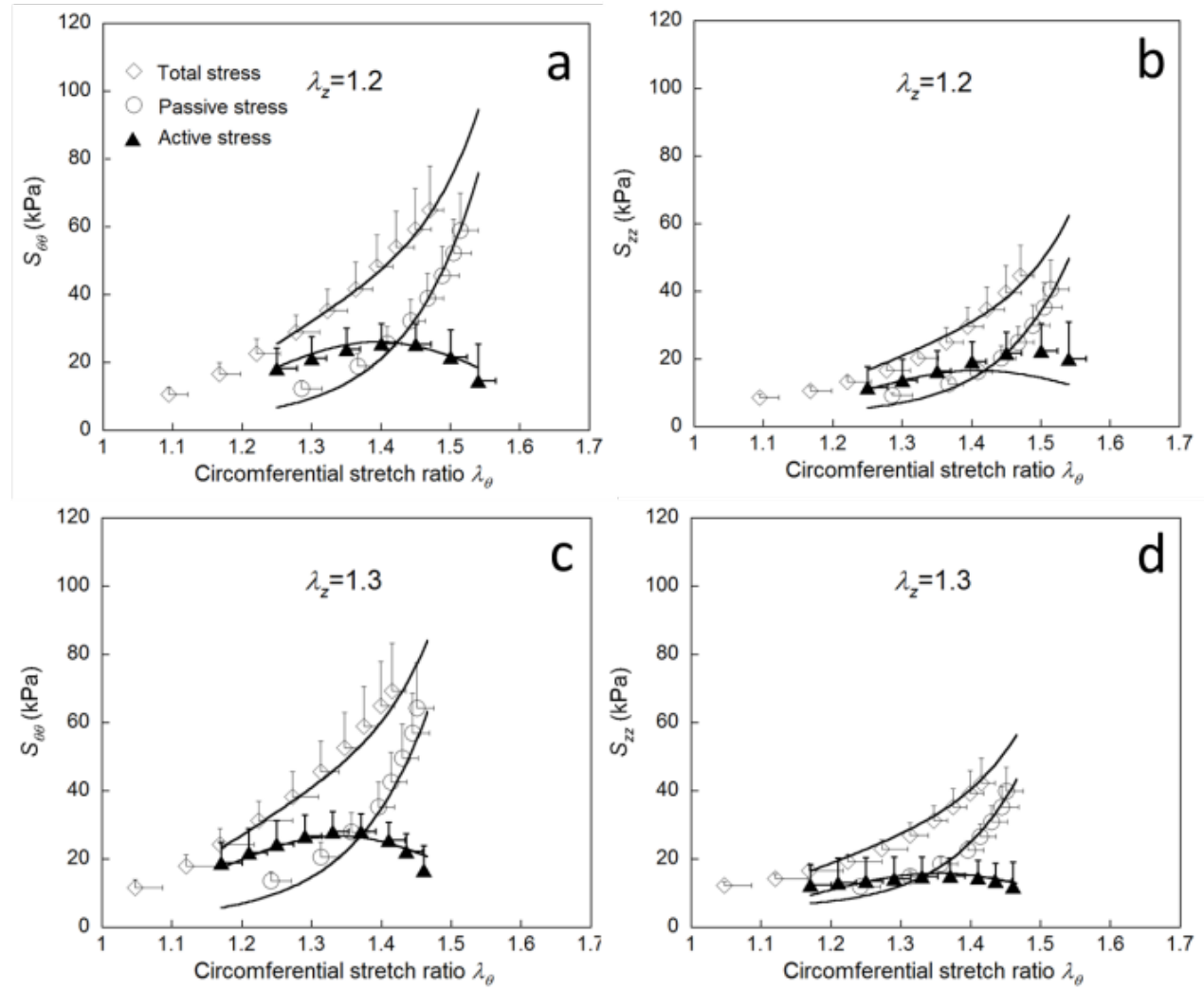

Figure 3. The total, passive and active stresses of coronary media. (a, b) The circumferential and axial stresses at $\lambda_{\mathrm{z}}=1.2$; (c, d) The circumferential and axial stresses at $\lambda_{\mathrm{z}}=1.3$. 
based model of active coronary media, which accounts for material properties of individual collagen and elastin fibers, and VSMCs. The measured VSMC orientation and distribution implies that VSMC constriction generates not only circumferential active stress, but also axial active responses in coronary arteries [11].

The nonlinear geometrical parameter-pressure relations of VSMC and nuclei, consistent with that of the outer diameter of vessels, suggest that recruited collagen fibers either in media or adventitia prevent the intact vessel as well as VSMC from overstretch at high pressure [12,15,20,21]. Additionally, the deformation of individual VSMC was found to be affine. VSMC connect with the extracellular matrix (ECM) via focal adhesion and the deformation thus strongly depends on ECM deformation as well as the macroscopic deformation of blood vessels. It is likely that flexible actin filaments deform with ECM through dense bodies in passive tissue such that collagen and elastin fibers follow affine deformation [22,23]. VSMC become stiffer during vasoconstriction due to the forces generated by actin-myosin interaction and tensile properties of cytoskeletal filaments increase significantly in contraction. Hence, the affine deformation assumption needs to be directly tested in active VSMC. Moreover, the elongation of the nuclei suggested that the tension developed by the cytoskeleton is transferred to the nuclei which may influence gene transcription and cellular phenotypes [14,24]. Consequently, the determination of strain and stress on individual VSMC is essential for better understanding of VSMC functions in normal and diseased arteries. This requires the development of microstructure based models to accurately predict the micro-environment of cells and nuclei.

The biaxial vasoactiviy of blood vessels were found in many studies. Huo et al. found that the porcine coronary artery displayed significant biaxial vasoconstriction induced by a K+ physiological saline solution [2,3]. Lu and Kassab [1] observed that there were significant axial force changes during vasomotions of carotid and femoral arteries, and Hayman et al showed that VSMC vasoconstriction reduced artery buckling as compared with relaxed conditions [5], indicating that vasoactivity may shorten the artery in the axial direction. These studies suggest that the biaxial vasoactivity of arteries is related to the helical structure of VSMC in muscular arteries. When assuming a simple one-dimension constitutive law for active VSMCs, the ratio of active axial to circumferential stresses was predicted as 0.12 for any axial stretch ratio, which was significantly lower than experimental measurements $\approx 0.6)$. It suggests that there exists mul ti-axial VSMC vasoconstriction in coronary media. The axial active response was principally induced by the multi-axial contraction of VSMC, denoted by the mean ratio of transversal to axial active stress of a single muscle fiber (0.4), while the oblique VSMC arrangement contributed about $30 \%$. With the influence of helical orientation of VSMC, the larger axial stretch ratio $\lambda_{z}=1.3$ further stretches the oblique VSMC and the peak active stresses, thus occurs earlier than that of $\lambda_{z}=1.2$. The present micro-structural model, based on a structural passive model and a two-dimensional model of active VSMC [2,3], can accurately predict the biaxial vasoactivity of coronary media based on the measured microstructure [11].

Some limitations need to be mentioned. First, a threedimensional constitutive model should be developed for individual active VSMCs, and a three-dimensional micro-structural model for vessels can be further proposed. Second, the present model does not account for the VSMCs in the lamella adjacent to intima or adventitia, where VSMC aligned towards the axial direction $[14,25]$ and may contribute partially to the biaxial active response of blood vessels. Finally, a microstructure-based model of the entire vessel (adventitia and media), should be integrated to investigate macro- and micro-scopic mechanical behaviors of active vessels $[3,26]$.

In summary, the present study provided morphological data of VSMCs of coronary media, based on which an active biaxial microstructure-based constitutive model was developed. The micro-structural model can accurately predict the biaxial mechanical responses of coronary media, and provides a more accurate framework for the biomechanics of blood vessels.

\section{REFERENCES}

[1] X. Lu and G. S. Kassab, "Vasoactivity of Blood Vessels using A Novel Isovolumic Myograph,” Annals of Biomedical Engineering, Vol. 35, 2007, pp. 356-366. http://dx.doi.org/10.1007/s10439-006-9243-0

[2] Y. Huo, Y. Cheng, X. Zhao, X. Lu and G. S. Kassab, "Biaxial Vasoactivity of Porcine Coronary Artery," American Journal of Physiology Heart and Circulatory Physiology, Vol. 302, 2012, pp. 2058-2063. http://dx.doi.org/10.1152/ajpheart.00758.2011

[3] Y. Huo, X. Zhao, Y. Cheng, X. Lu and G. S. Kassab, "Two-Layer Model of Coronary Artery Vasoactivity," Journal of Applied Physiology, Vol. 114, 2013, pp. 14511459. http://dx.doi.org/10.1152/japplphysiol.01237.2012

[4] M. A. Gaballa, C. T. Jacob, T. E. Raya, J. Liu, B. Simon and S. Goldman, "Large Artery Remodeling During Aging: Biaxial Passive and Active Stiffness," Hypertension, Vol. 32, No. 3, 1998, pp. 437-443.

http://dx.doi.org/10.1161/01.HYP.32.3.437

[5] D. M. Hayman, J. Zhang, Q. Liu, Y. Xiao and H. C. Han, "Smooth Muscle Cell Contraction Increases the Critical Buckling Pressure of Arteries,” Journal of Biomechanics, Vol. 46, 2013, pp. 841-844.

http://dx.doi.org/10.1016/j.jbiomech.2012.11.040 
[6] H. Wolinsky and S. Glagov, "A Lamellar Unit of Aortic Medial Structure and Function in Mammals," Circulation Research, Vol. 20, 1967, pp. 99-111. http://dx.doi.org/10.1161/01.RES.20.1.99

[7] M. J. Osborne-Pellegrin. "Some Ultrastructural Characteristics of the Renal Artery and Abdominal Aorta in the Rat,” Journal of Anatomy, Vol. 125, 1978, pp. 641-652.

[8] J. A. G. Rhodin. “Architecture of the Vessel Wall,” In: D. F. Bohr, A. P. Somlyo, A. V. Sparks, Eds., Handbook of Physiology, Section 2: The Cardiovascular System, Volume II, American physiology society, Bethesda, 1980, pp. $1-31$.

[9] T. Fujiwara and Y. Uehara, "The Cytoarchitecture of the Medial Layer in Rat Thoracic Aorta: A Scanning Electron-microscopic Study,” Cell and Tissue Research, Vol. 270, 1992, pp. 165-172. http://dx.doi.org/10.1007/BF00381891

[10] J. Stålhand, A. Klarbring and G. A. Holzapfel, “A Mechanochemical 3D Continuum Model for Smooth Muscle Contraction under Finite Strains,” Journal of Theoretical Biology, Vol. 268, 2011, pp. 120-130. http://dx.doi.org/10.1016/j.jtbi.2010.10.008

[11] H. Chen, T. Luo, X. F. Zhao, X. Lu, Y. L. Huo and G. S. Kassab, "V Microstructural Constitutive Model of Active Coronary Media,” Biomaterials, Vol. 34, No. 31, 2013,pp. 7575-7583.

http://dx.doi.org/10.1016/j.biomaterials.2013.06.035

[12] J. M. Clark and S. Glagov, "Transmural Organization of the Arterial Media. The Lamellar Unit Revisited,” Arteriosclerosis, Vol. 5, 1985 pp. 19-34. http://dx.doi.org/10.1161/01.ATV.5.1.19

[13] T. R. Hansen, D. X. Dineen, G. L. Pullen, “Orientation of Arterial Smooth Muscle and Strength of Contraction of Aortic Strips from DOCA-hypertensive Rats,” Blood Vessels, Vol. 17, 1980, pp. 302-311.

[14] M. K. O’Connell, S. Murthy, S. Phan, C. Xu, J. Buchanan, R. Spilker, et al., "The Three-Dimensional Micro- and Nanostructure of the Aortic Medial Lamellar Unit Measured Using 3D Confocal and Electron Microscopy Imaging,” Matrix Biology, Vol. 27, 2008, pp. 171-181. http://dx.doi.org/10.1016/j.matbio.2007.10.008

[15] A. Zoumi, X. Lu, G. S. Kassab and B. J. Tromberg, "Imaging Coronary Artery Microstructure Using SecondHarmonic and Two-Photon Fluorescence Microscopy,” Biophysical Journal, Vol. 87, 2004, pp. 2778-2786. http://dx.doi.org/10.1529/biophysj.104.042887

[16] M. Y. Loqman, P. G. Bush, C. Farquharson, A. C. Hall, "A Cell Shrinkage Artefact in Growth Plate Chondrocytes with Common Fixative Solutions: Importance of Fixative Osmolarity for Maintaining Morphology,” European Cells and Materials, Vol. 19, 2010, pp. 214-227.
[17] Y. Hollander, D. Durban, X. Lu, G. S. Kassab and Y. Lanir, "Experimentally Validated Microstructural 3D Constitutive Model of Coronary Arterial Media,” Journal of Biomechanical Engineering, Vol. 133, 2011, p. 031007. http://dx.doi.org/10.1115/1.4003324

[18] Y. Hollander, D. Durban, X. Lu, G. S. Kassab and Y. Lanir, "Constitutive Modeling of Coronary Arterial Media-comparison of Three Model Classes," Journal of Biomechanical Engineering, Vol. 133, 2011, p. 061008. http://dx.doi.org/10.1115/1.4004249

[19] B. E. Carlson and T. W. Secomb, “A Theoretical Model for the Myogenic Response Based on the Length-tension Characteristics of Vascular Smooth Muscle," Microcirculation, Vol. 12, 2005, pp. 327-338. http://dx.doi.org/10.1080/10739680590934745

[20] H. Chen, Y. Liu, M. N. Slipchenko, X. F. Zhao, J. X. Cheng and G. S. Kassab, "The Layered Structure of Coronary Adventitia under Mechanical Load,” Biophysical Journal, Vol. 101, 2011, pp. 2555-2562. http://dx.doi.org/10.1016/j.bpj.2011.10.043

[21] H. Chen, X. Zhao, X. Lu and G. S. Kassab, "Non-linear Micromechanics of Soft Tissues," International Journal of Non-Linear Mechanics, Vol. 56, 2013, pp. 79-85. http://dx.doi.org/10.1016/j.ijnonlinmec.2013.03.002

[22] M. S. Sacks. "Incorporation of Experimentally-derived Fiber Orientation into A Structural Constitutive Model for Planar Collagenous Tissues,” Journal of Biomechanical Engineering, Vol. 125, 2003, pp. 280-287. http://dx.doi.org/10.1115/1.1544508

[23] J. A. Stella, J. Liao, Y. Hong, W. David Merryman, W. R. Wagner and M. S. Sacks, "Tissue-to-cellular Level Deformation Coupling in Cell Micro-integrated Elastomeric Scaffolds,” Biomaterials, Vol. 29, 2008, pp. 3228-3236. http://dx.doi.org/10.1016/j.biomaterials.2008.04.029

[24] D. E. Ingber, “Cellular Mechanotransduction: Putting All the Pieces Together Again,” The FASEB Journal, Vol. 20, 2006, pp. 811-827. http://dx.doi.org/10.1096/fj.05-5424rev

[25] L. H. Timmins, Q. Wu, A. T. Yeh and J. E. Moore, "Greenwald SE. Structural Inhomogeneity and Fiber Orientation in the Inner Arterial Media," American Journal of Physiology Heart and Circulatory Physiology, Vol. 298, 2010, pp. 1537-1545. http://dx.doi.org/10.1152/ajpheart.00891.2009

[26] S. Verlohren, G. Dubrovska, S. Y. Tsang, K. Essin, F. C. Luft, Y. Huang, et al., "Visceral Periadventitial Adipose Tissue Regulates Arterial Tone of Mesenteric Arteries," Hypertension, Vol. 44, 2004, pp. 271-276. http://dx.doi.org/10.1161/01.HYP.0000140058.28994.ec 